Published in final edited form as:

Cancer Epidemiol Biomarkers Prev. 2010 February ; 19(2): 443-446. doi:

10.1158/1055-9965.EPI-09-0930.

\title{
Prevalence of tamoxifen use for breast cancer chemoprevention among U.S. women
}

\author{
Erika A. Waters ${ }^{1}$, Kathleen A. Cronin², Barry I. Graubard ${ }^{3}$, Paul K. Han ${ }^{2}$, and Andrew N. \\ Freedman ${ }^{2}$ \\ ${ }^{1}$ Cancer Prevention Fellowship Program, Center for Cancer Training, Health Communication and \\ Informatics Research Branch/Division of Cancer Control and Population Sciences, National \\ Cancer Institute, Bethesda, MD \\ ${ }^{2}$ Division of Cancer Control and Population Sciences, National Cancer Institute, Bethesda, MD \\ ${ }^{3}$ Division of Cancer Epidemiology and Genetics, National Cancer Institute, Bethesda, MD
}

\begin{abstract}
Background-Tamoxifen can reduce the risk of developing invasive estrogen-receptor positive (ER+) breast cancer by $49 \%$, but it is unknown how many women in the U.S. are taking tamoxifen for primary prevention of breast cancer.
\end{abstract}

Methods-Data from the years 2000 and 2005 National Health Interview Surveys (NHIS) were analyzed to estimate the prevalence of tamoxifen use among U.S. women for primary chemoprevention of breast cancer.

Results-In 2000, approximately 0.2 percent of U.S. women aged 40 to 79 without a personal history of breast cancer took tamoxifen for chemoprevention (95\% CI 0.13-0.31). In 2005, the prevalence was approximately $0.08 \%$ (95\% CI 0.03-0.17).

Conclusion-The prevalence of tamoxifen use for primary prevention of breast cancer was very low in the years 2000 and 2005. Possible explanations for the low uptake are explored.

\section{Keywords}

Chemoprevention; tamoxifen; side effect

\begin{abstract}
In 1998 the Breast Cancer Prevention Trial (BCPT), a large prospective randomized control trial, demonstrated that tamoxifen can reduce the risk of invasive estrogen-receptor positive $(\mathrm{ER}+)$ breast cancer in high-risk women by 49 percent (1). Tamoxifen also appeared to protect against hip fractures, but it increased the risk of endometrial cancer, stroke, pulmonary embolism, and deep-vein thrombosis. These findings were consistent with the results of other chemoprevention trials (2). In 1999 the U.S. Food and Drug Administration (FDA) approved tamoxifen for primary prevention of breast cancer.
\end{abstract}

Despite adverse effects, using tamoxifen for primary prevention of breast cancer may be appropriate and beneficial for some high-risk women (3). Data from the year 2000 National Health Interview Survey (NHIS) suggest that although 10 million U.S. women aged 35-79 years would be eligible for tamoxifen chemoprevention based on their risk factors, for 2.4 million of these women the benefits of taking tamoxifen would outweigh the risks (4).

Reprint requests should be addressed to: Erika A. Waters, Executive Plaza North Room 4051B, 6130 Executive Blvd MSC 7365 , Bethesda, MD 20892; erika.a.waters@gmail.com. 
It has been over ten years since the first results from BCPT were published and tamoxifen was approved by the FDA for breast cancer chemoprevention. It is reasonable to conclude that, in the intervening years, as physicians and patients have become more aware of the availability of using tamoxifen to reduce the risk of breast cancer in high-risk women, the use of this drug would increase for this indication. In the present study we report the prevalence of tamoxifen use for breast cancer chemoprevention among a nationally representative sample of U.S. women in the years 2000 and 2005. We then draw upon the existing clinical and psychological literatures to explore possible explanations for our findings.

\section{Methods}

We used NHIS data from 2000 and 2005 to estimate the number of women taking tamoxifen for primary chemoprevention of breast cancer. Tamoxifen use was attributed to breast chemoprevention if the participant indicated that she had never been diagnosed with breast cancer. Details describing the survey design and sampling frames have been described previously (5).

Prevalence estimates, including totals and percentages, were weighted by the NHIS sample weights to the total U.S. population. The denominator included all women in the U.S. aged 40 to 79 who did not have a history of breast cancer. Standard errors used in computing the 95\% confidence intervals (CIs) were estimated to take into account the complex multistage probability sampling design of the NHIS (6). We calculated CIs for small percentages using a modified binomial CI (7). All calculations were performed using SAS version 9.1.3 for Linux and SUDAAN version 10.0.1 software $(8,9)$.

\section{Results}

The prevalence of tamoxifen use in 2000 and 2005 was very low (see Table 1). In 2000, approximately $0.2 \%(95 \%$ CI $0.13-0.31)$ or 120,737 (95\% CI 75,416-183,219) of U.S. women aged 40 to 79 without a personal history of breast cancer took tamoxifen for chemoprevention. In 2005, the prevalence was approximately $0.08 \%$ (95\% CI 0.03-0.17) and population size was 51,575 (95\% CI 19,596-109,936).

Additional informal analyses revealed that, of the 35 women in the sample who took tamoxifen in either 2000 or 2005, 7 (in 2000) and 4 (in 2005) had Gail scores indicative of being eligible for tamoxifen chemoprevention (i.e., estimated probability of being diagnosed with breast cancer in the next five years at or above 1.67\%). Conversely, 20 (in 2000) and 4 (in 2005) had Gail scores that were below the criterion for tamoxifen eligibility. However, our analysis of the Gail model did not include information about atypical hyperplasia because NHIS 2000 and 2005 did not ask women to report whether or not they had ever received such a diagnosis. Therefore, the analysis may have underestimated women's breast cancer risk. Neither does the analysis account for having a history of breast cancer on the paternal side of the family or by cancer among second-degree relatives, such as a grandmother or aunts. This makes it difficult to determine whether the low Gail scores among some women who were taking tamoxifen for chemoprevention were indicative of actual low risk or whether the score did not represent their actual risk because they had risk factors that were not accounted for by the model.

\section{Discussion}

This study found that the prevalence of tamoxifen use by U.S. women for chemoprevention was exceptionally low. In the year after tamoxifen was approved for chemoprevention of 
breast cancer, less than one quarter of one percent of women aged 40 to 79 without a history of breast cancer were taking it, and this number decreased slightly (but not significantly) over time. These results, in conjunction with other research findings (4), suggest that very few women who would be eligible to take tamoxifen for chemoprevention are doing so.

One possible explanation for the low uptake in tamoxifen is a reluctance of physicians to prescribe it. Family physicians in one study advised patients against using tamoxifen in $77 \%$ of cases (10), even though the women were at high risk and had been evaluated for a breast lump. Another study found that surgeons were much less willing than oncologists to prescribe the drug (11). This discrepancy was attributed to differential focus on treatment outcomes. For example, oncologists were more concerned about recurrence of breast cancer, but surgeons were more concerned about the increased risk of cataracts. Physician education about breast cancer risk reduction techniques might also be associated with their willingness to prescribe tamoxifen. One study found that physicians who felt insufficiently informed about risk reduction options and/or in risk reduction counseling were less than half as likely to prescribe tamoxifen or raloxifene than physicians who felt that they were sufficiently informed and trained (12). Future research might investigate the effects of physician education on cancer prevention counseling, including chemopreventive agents like tamoxifen.

Low uptake of tamoxifen might also be attributable to a reluctance of patients to take it. When women at high risk of breast cancer are offered tamoxifen, few accept treatment. For example, in one small study only 2 of 43 eligible women began tamoxifen therapy (13). In another study, only 1 of the 89 high-risk women who were offered tamoxifen accepted it, even though all 89 had undergone recent biopsies for breast lumps (10). In two chemoprevention trials that included tamoxifen as a treatment arm, the proportion of eligible women who agreed to participate was less than $25 \%$ (1) (25), possibly due to concern about the side effects.

Patients' reluctance to take tamoxifen might originate from various sources. One possibility is that taking hormone replacement therapy (HRT) is contraindicated with tamoxifen use. Women who take HRT to alleviate debilitating hot flashes may not be willing to discontinue HRT in order to reduce their risk of breast cancer, and physicians may or may not agree with these decisions. This possible conflict makes HRT an important aspect of the clinical decision process for both doctors and patients. A second possibility is that the patient's health coverage might not cover the cost of tamoxifen for primary chemoprevention.

Another explanation could be that some patients believe that the benefits of taking tamoxifen do not outweigh the risks. Making medical decisions is extraordinarily complex because medical treatments often have multiple benefits and multiple side effects that vary in severity. Calculating how these benefits and risks combine to affect overall health is very difficult. However, many people do not understand how to combine probabilistic risk and benefit information even when a treatment has only one benefit and only one risk (14). These individuals would likely experience great difficulty evaluating the overall effects of chemopreventive therapy. Nevertheless, a lack of comprehension or education is not sufficient to explain reluctance to accept chemopreventive therapy (14-16). Instead, merely suggesting that a treatment has a side effect or a surgical complication can be highly aversive $(17,18)$. Qualitative studies suggest that some women might perceive the risks of side effects as more probable and as more dangerous than the risks of breast cancer $(19,20)$. Informing women how their risk of breast cancer compares to the risk of other women $(14,21)$, displaying the risk information as arrays of icons or tables $(14,16,22,23)$, or highlighting the number of people saved by treatment (24) may address this problem. However, for some women, side effects that affect quality of life (e.g., hot flashes, sexual 
functioning) hold more importance than their risk of breast cancer (20). Consequently, communicating the risks and benefits of treatment should be performed carefully to avoid undermining patient autonomy.

It might be argued that low acceptance of tamoxifen is due to concerns about the increased risk of endometrial cancer. If this is the case, chemopreventive agents like raloxifene, which has a more favorable side effect profile than tamoxifen (25), should be more acceptable to patients and physicians. Approximately 530,000 women were taking raloxifene in 2005 (analyses not shown), but it is unknown how many of these women were using it for chemoprevention and how many were using it to treat osteoporosis. The FDA approved raloxifene for breast cancer chemoprevention in 2007. However, to the best of our knowledge, no studies have examined how women perceive its risks and benefits. We would speculate that many women would be reassured by the reduced risk of endometrial cancer associated with raloxifene compared with tamoxifen (25). Nevertheless, we also hypothesize that concerns about side effects that raloxifene shares with tamoxifen—such as hot flashes, sexual dysfunction, and pulmonary embolism - would still lead some women to decline treatment (15). Qualitative and quantitative research should examine whether and how women's beliefs about raloxifene differ from their beliefs about tamoxifen, as well as how physicians perceive using tamoxifen and raloxifene for primary prevention. Additional strategies for communicating the risks and benefits of chemopreventive treatments should be developed, but these strategies should not impinge on patients' autonomy or their ability to make an informed decision (e.g., by presenting data using risk communication formats that are not easily understood).

These analyses are limited by two main factors. First, the number of women taking tamoxifen for chemoprevention was extremely low-27 in the year 2000 and 8 in the year 2005. These small numbers led to large standard errors and wide confidence intervals in the prevalence estimates. Nevertheless, it is reasonable to conclude that prevalence of tamoxifen use for chemoprevention in the U.S. is well below 1\%. The second limitation is that the data are self-reported. Women might be reluctant to report their medication use, be unaware of the purpose of taking tamoxifen, or be unaware of the name of the medication. They may also simply forget they are taking it. All of these factors would result in an underestimation of the prevalence of tamoxifen use. However, even if the actual prevalence is double that of the estimates presented in this brief report, the overall prevalence in the U.S. would not exceed $1 \%$. More minor limitations include the absence of BRCA1/BRCA2 risk status questions in the NHIS and the inability to examine prevalence stratified by risk status due to extremely small cell sizes.

We found that the prevalence of tamoxifen use in U.S. women was low in 2000 and in 2005. These data, combined with previous research that estimated the number of women who might benefit from chemopreventive therapy for breast cancer (4), suggest that few women are taking the drug. Breast cancer chemoprevention is not appropriate for all eligible high risk women, despite the availability of statistical models to calculate the likelihood of benefiting from tamoxifen. For each individual woman, the benefits of chemoprevention breast cancer treatment must be weighed carefully against its risks and decision-making should incorporate the many medical, psychosocial and personal factors that are unique to that woman $(3,15)$.

\section{Acknowledgments}

Financial Support: Erika Waters was supported by the National Cancer Institute's Cancer Prevention Fellowship Program. 


\section{References}

1. Fisher B, Constantino JP, Wickerham DL, et al. Tamoxifen for prevention of breast cancer: Report of the National Surgical Adjuvant Breast and Bowel Project P-1 Study. J Natl Cancer Inst 1998;90:1371-88. [PubMed: 9747868]

2. Cuzick J, Powles T, Veronesi U, et al. Overview of the main outcomes in breast-cancer prevention trials. Lancet 2003;361:296-300. [PubMed: 12559863]

3. Gail MH, Costantino JP, Bryant J, et al. Weighing the risks and benefits of tamoxifen treatment for preventing breast cancer. J Natl Cancer Inst 1999;91:1829_46. [PubMed: 10547390]

4. Freedman AN, Graubard BI, Rao SR, McCaskill-Stevens W, Ballard-Barbash R, Gail MH. Estimates of the number of U.S. women who could benefit from tamoxifen for breast cancer chemoprevention. J Natl Cancer Inst 2003;95:526-32. [PubMed: 12671020]

5. CDC/National Center for Health Statistics [Internet]. About the National Health Interview Survey. [updated 2009 June 17]. [cited 2009 June 30]. Available from: http://www.cdc.gov/nchs/nhis/about_nhis.htm

6. Botman SL, Moore TF, Moriarty CL, Parsons VL. Design and estimation for the National Health Interview Survey, 1995-2000. Natl Vital Stat Rep 2000:1-31.

7. Korn, EL.; Graubarrd, BI. Analysis of health surveys. John Wiley \& Sons; New York: 1999. p. 22-28.p. 64-8.

8. SAS Institute Inc.. SAS v. 9.1.3 for Linux. SAS Institute; Cary, NC: 2002-2003.

9. Research Triangle Institute. SUDAAN v. 10.0.1. Research Triangle Park, NC: 2009.

10. Taylor R, Taguchi K. Tamoxifen for breast cancer chemoprevention: Low uptake by high-risk women after evaluation of a breast lump. Ann Fam Med 2005;3:242-7. [PubMed: 15928228]

11. Malek K, Fink AK, Thwin SS, Gurwitz J, Ganz PA, Silliman RA. The relationship among physicians' specialty, perceptions of the risks and benefits of adjuvant tamoxifen therapy, and its recommendation in older patients with breast cancer. Med Care 2004;42:700-6. [PubMed: 15213495]

12. Kaplan CP, Haas JS, Pérez-Stable EJ, Des Jarlais G, Gregorich SE. Factors affecting breast cancer risk reduction practices among California physicians. Prev Med 2005;41:7-15. [PubMed: 15916987]

13. Port ER, Montgomery LL, Heerdt AS, Borgen PI. Patient reluctance toward tamoxifen use for breast cancer primary prevention. Ann Surg Oncol 2001;8:580-5. [PubMed: 11508619]

14. Waters EA, Weinstein ND, Colditz GA, Emmons K. Reducing aversion to side effects in preventive medical treatment decisions. J Exp Psychol Appl 2007;13:11-21. [PubMed: 17385998]

15. Melnikow J, Paterniti D, Azari R, et al. Preferences of women evaluating risks of tamoxifen (POWER) study of preferences for tamoxifen for breast cancer risk reduction. Cancer 2005;103:1996-2005. [PubMed: 15825209]

16. Schwartz LM, Woloshin S, Welch HG. Using a drug facts box to communicate drug benefits and harms. Ann Intern Med 2009;150:516-27. [PubMed: 19221371]

17. Waters EA, Weinstein ND, Colditz GA, Emmons K. Explanations for side effect aversion in preventive medical treatment decisions. Health Psychol 2009;28:201-9. [PubMed: 19290712]

18. Amsterlaw J, Zikmund-Fisher BJ, Fagerlin A, Ubel PA. Can avoidance of complications lead to biased healthcare decisions? Judgm Decis Mak 2006;1:64-75.

19. Heisey R, Pimlott N, Clemons M, Cummings S, Drummond N. Women's views on chemoprevention of breast cancer. Can Fam Physician 2006;52:624-5. [PubMed: 17327893]

20. Paterniti DA, Melnikow J, Henderson S, DeGregorio M, Kupperman M, Nease R. "I'm going to die of something anyway": Women's perceptions of tamoxifen for breast cancer risk reduction. Ethn Dis 2005;15:365-72. [PubMed: 16108294]

21. Fagerlin A, Zikmund-Fisher BJ, Ubel PA. "If I'm better than average, then I'm ok?": Comparative information influences beliefs about risk and benefits. Patient Educ Couns 2007;69:140-4. [PubMed: 17942271]

22. Hawley ST, Zikmund-Fisher BJ, Ubel P, Jancovic A, Lucas T, Fagerlin A. The impact of the format of graphical presentation on health-related knowledge and treatment choices. Patient Educ Couns 2008;73:448-55. [PubMed: 18755566] 
23. Zikmund-Fisher BJ, Ubel PA, Smith DM, et al. Communicating side effect risks in a tamoxifen prophylaxis decision aid: The debiasing influence of pictographs. Patient Educ Couns 2008;73:209-14. [PubMed: 18602242]

24. Zikmund-Fisher BJ, Fagerlin A, Roberts TR, Derry HA, Ubel PA. Alternate methods of framing information about medication side effects: Incremental risk versus total risk of occurrence. J Health Commun 2008;13:107-24. [PubMed: 18300064]

25. Vogel VG, Costantino JP, Wickerham DL, et al. Effects of tamoxifen vs raloxifene on the risk of developing invasive breast cancer and other disease outcomes: The NSABP study of tamoxifen and raloxifene (STAR) P-2 trial. JAMA 2006;295:2727-841. [PubMed: 16754727] 
Table 1

Prevalence estimates for use of tamoxifen in two nationally-representative U.S. data sources (NHIS 2000, NHIS 2005)

\begin{tabular}{|c|c|c|c|}
\hline \multicolumn{2}{|l|}{ NHIS $2000^{I}$} & \multicolumn{2}{|l|}{ NHIS $2005^{2}$} \\
\hline $\begin{array}{l}\text { Number using } \\
\text { tamoxifen } \\
(95 \% \text { CI })\end{array}$ & Percentage & $\begin{array}{l}\text { Number using } \\
\text { tamoxifen } \\
(95 \% \text { CI })\end{array}$ & Percentage \\
\hline $\begin{array}{l}120,737 \\
(95 \% \text { CI } 75,416-183,219)\end{array}$ & $\begin{array}{l}0.20 \\
(95 \% \text { CI } 0.13-0.31)\end{array}$ & $\begin{array}{l}51,575 \\
(95 \% \text { CI } 19,596-109,936)\end{array}$ & $\begin{array}{l}0.08 \\
(95 \% \text { CI } 0.03-0.17)\end{array}$ \\
\hline
\end{tabular}

${ }^{1}$ Weighted estimate from a sample of 27 women who report using tamoxifen out of a total sample size of 10,601

${ }^{2}$ Weighted estimate from a sample of 8 women who report using tamoxifen out of a total sample size of 10,690 